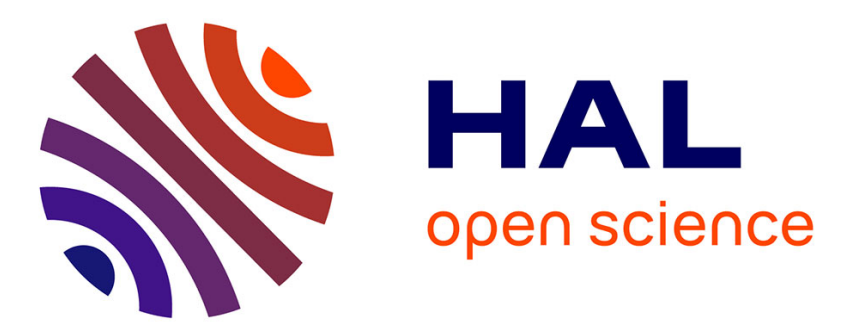

\title{
Automatic classification of skin lesions using geometrical measurements of adaptive neighborhoods and local binary patterns
}

Victor González-Castro, Johan Debayle, Yanal Wazaefi, Mehdi Rahim, Caroline Gaudy, Jean-Jacques Grob, Bernard Fertil

\section{To cite this version:}

Victor González-Castro, Johan Debayle, Yanal Wazaefi, Mehdi Rahim, Caroline Gaudy, et al.. Automatic classification of skin lesions using geometrical measurements of adaptive neighborhoods and local binary patterns. ICIP 2015 IEEE International Conference on Image Processing, IEEE Signal Processing Society, Sep 2015, Québec City, Canada. pp.1722 à 1726, 10.1109/ICIP.2015.7351095 . emse-01226873

\section{HAL Id: emse-01226873 \\ https://hal-emse.ccsd.cnrs.fr/emse-01226873}

Submitted on 10 Nov 2015

HAL is a multi-disciplinary open access archive for the deposit and dissemination of scientific research documents, whether they are published or not. The documents may come from teaching and research institutions in France or abroad, or from public or private research centers.
L'archive ouverte pluridisciplinaire HAL, est destinée au dépôt et à la diffusion de documents scientifiques de niveau recherche, publiés ou non, émanant des établissements d'enseignement et de recherche français ou étrangers, des laboratoires publics ou privés. 


\title{
AUTOMATIC CLASSIFICATION OF SKIN LESIONS USING GEOMETRICAL MEASUREMENTS OF ADAPTIVE NEIGHBORHOODS AND LOCAL BINARY PATTERNS
}

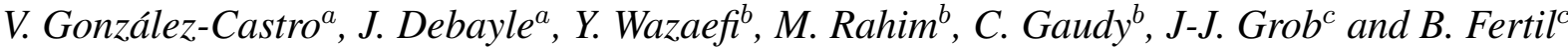 \\ ${ }^{a}$ École Nat. Sup. des Mines de Saint-Étienne, LGF UMR CNRS 5307, Saint-Étienne, France \\ ${ }^{b}$ Lab. des Sciences de l'Information et des Systems, UMR CNRS 7296, Marseille, France \\ ${ }^{c}$ Service de Dermatologie, Hôpital de la Timone, Marseille, France \\ Corresponding author: J. Debayle / debayle@emse. fr
}

\begin{abstract}
This paper introduces a method for characterizing and classifying skin lesions in dermoscopic color images with the goal of detecting which ones are melanoma (cancerous lesions). The images are described by means of the Local Binary Patterns (LBPs) computed on geometrical feature maps of each color component of the image. These maps are extracted from geometrical measurements of the General Adaptive Neighborhoods (GAN) of the pixels. The GAN of a pixel is a region surrounding it and fitting its local image spatial structure. The performance of the proposed texture descriptor has been evaluated by means of an Artificial Neural Network, and it has been compared with the classical LBPs. Experimental results using ROC curves show that the GAN-based method outperforms the classical one and the dermatologists' predictions.
\end{abstract}

Index Terms - Melanoma, Texture description, General adaptive neighborhoods, Local binary patterns

\section{INTRODUCTION}

In 2012 there were more than 11150 new cases of skin cancer in France (3.1\% of all detected cancers), $15 \%$ of which were mortal. Late diagnosis of skin cancer makes treatments much less efficient (i.e. a melanoma may become very aggressive in just a few months). Therefore, its early detection becomes essential to improve the chances of curing the skin cancer and, thus, the survival chances of the patient.

This is not an easy task for a non-experienced observer, as it is evidenced by the example shown in figure 1. To carry out the detection of melanoma, dermatologists use several state-of-the-art methods, often called rules, such as the ABCD rule [1] (Asymmetry, Border irregularity, Color irregularity and Differential structure, i.e. the size and number of structural features), the Menzies scoring or the 7-point checklist [2], which are based on the presence of certain texture patterns. Dermatologists detect malignancy features based on these rules and then combine this information to predict

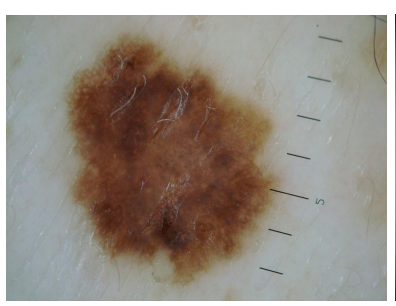

(a) benign lesion

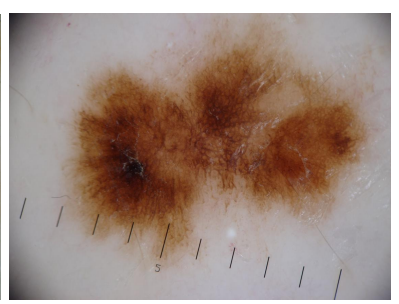

(b) melanoma
Fig. 1. Example of an image of a benign lesion (a) and a melanoma (b).

malignancy of the tumor. An automation of such individual analysis of nevi would be very helpful not only for dermatologists, as they would make more accurate diagnosis and, thus, make better decisions about the need of surgery, but also for general practitioners, sending patients to the specialist more appropriately. Therefore, it would save economic and time resources to the Social Security system.

Automatic melanoma detection - based on the analysis of dermoscopy images - has been receiving an increasing attention in the literature [3]. Most works reproduce the classic rules used by dermatologists, extracting features linked to them (see for instance [4]). However, the extraction of such kind of features needs a previous segmentation of the lesion, which is often considered a very tricky step, as it may not be clear, even for specialists, where the boundaries of the lesion are. Other methods have been investigated in the literature where dermoscopic images do not need any segmentation process as proposed in [5]. Such a method uses a texture descriptor computed with the Local Binary Patterns $[6,7,8]$ (LBPs). Nethertheless, only intensity information of the color components are taken into consideration.

In this paper an automatic classification method of skin lesions from dermoscopic images is proposed by combining LBPs and local geometrical measurements. The lesions are described by means of the LBPs from maps created by means of geometrical features of the General Adaptive Neighbor- 
hoods (GANs) [9] of pixels, as it is explained in detail in section 2. The performance of this texture descriptor has been assessed on a real dataset of skin lesions, as stated in section 3. Finally, conclusions and future perspectives are shown in section 4.

\section{THE PROPOSED TEXTURE DESCRIPTOR}

\subsection{General Adaptive Neighborhoods (GANs)}

The GANIP approach [9, 10] provides a general framework for multiscale, local and adaptive image processing and analysis of gray-level images. It is based on extracting spatial neighborhoods called General Adaptive Neighborhoods (GANs) from the points of the image, whose size and shape are adapted to the local features of the image. Specifically, a GAN is a subset of the spatial support $D \subseteq \mathbb{R}^{2}$ constituted by connected points whose values in relation to a selected criterion (luminance, contrast, etc.) fit within a homogeneity tolerance. As a result, GANs are adaptive with the spatial structures and self-defined from the image.

Let $f$ be an image defined in $D$ with range in $\mathbb{R}$, and let $h$ be a criterion mapping, also defined in $D$ and valued in $\mathbb{R}$, based on local measurements such as luminance, contrast, etc. For each point $x \in D$, the GANs (denoted $V_{m}^{h}(x)$ ) are subsets in $D$ built upon $h$ in relation to a homogeneity tolerance $m \in \mathbb{R}^{+}$. More precisely, $V_{m}^{h}(x)$ fulfills two conditions: 1 ) its points are close to $x$ in relation to the criterion mapping and 2) the GAN is a path-connected set.

Thus, the GANs are formally defined as:

$$
V_{m}^{h}(x)=C_{\{y \in D:|h(y)-h(x)| \leq m\}}(x)
$$

where $C_{X}(x)$ denotes the path connected component of $X \subseteq$ $D$ containing $x \in D$. Therefore, it is ensured that $\forall x \in$ $D \quad x \in V_{m}^{h}(x)$.

Figure 2 shows the GAN computed for one pixel of a dermoscopic color image using the luminance criterion of the red color component and the homogeneity tolerance $m=20$.

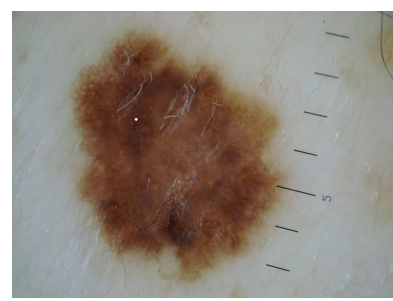

(a) original image with one selected pixel $x$

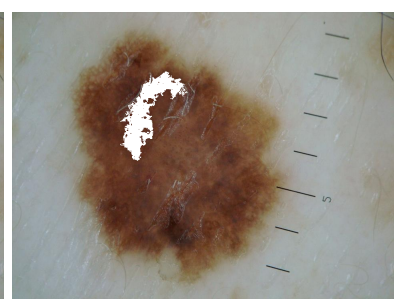

(b) GAN of the pixel $x$
Fig. 2. The GAN (b) of one pixel (a) of a dermoscopic image computed on the red color component, using the luminance criterion with the homogeneity tolerance value $m=20$.

The reader interested in further theoretical aspects on GANs is referred to [9].

\subsection{GAN-based Minkowski functionals}

Integral geometry provides a suitable family of geometrical and topological descriptors of 2-D and 3-D spatial patterns, known as Minkowski functionals [11]. In 2-D, there are three Minkowski functionals: area, perimeter and Euler number, denoted respectively $A, P$ and $\chi$.

These functionals are defined on the class of nonempty compact convex sets in $\mathbb{R}^{2}$. They have been extended to the convex ring [12], i.e. the set of all finite unions of convex bodies, which may be considered as a realistic Euclidean model for digital images.

The GAN-based Minkowski maps [13] are defined by assigning to each point $x \in D$ the Minkowski density functional of its GAN $V_{m}^{h}(x)$. More explicitly, the GAN-based Minkowski map of a gray-level image, denoted by $\mu_{m}^{h}$, is defined by:

$$
\mu_{m}^{h}(x)=\mu\left(V_{m}^{h}(x)\right)
$$

where $\mu$ denotes a Minkowski density functional (i.e. $\mu \equiv A$, $\mu \equiv P$ or $\mu \equiv \chi)$.

Figure 3 shows an example of GAN-based Minkowski map of an image using the area as functional. The GANs are homogeneous with respect to the luminance of the blue color component using the tolerance $m=20$. Therefore, the value at each point $x$ of the Minkowski map is the area of the GAN $V_{20}^{h}(x)$.

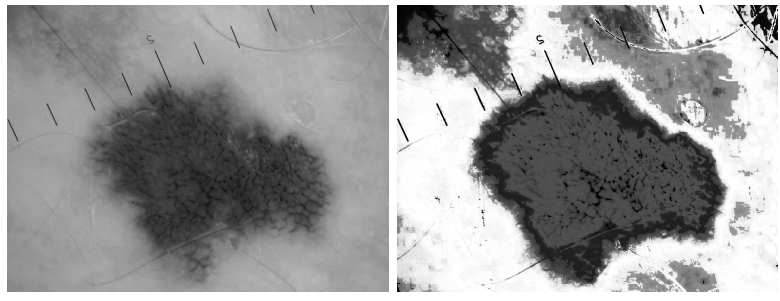

(a) blue component of an original image

(b) GAN-based area map

Fig. 3. The GAN-based area map (b) of the blue component (a) of a dermoscopic image computed with the luminance criterion and the homogeneity tolerance value $m=20$.

\subsection{Local Binary Patterns}

Local Binary Patterns were first introduced by Ojala et al. [6]. This original version worked in a $3 \times 3$ pixel block of an image, and were later generalized [7]. This generalization does not limit the size of the neighbor nor the number of sampling points.

Consider an image $I$, and let $g_{c}$ denote the gray level of a pixel $c$ in the coordinates $(x, y)$, i.e. $g_{c}=I(x, y)$. Let $g_{p}$ be the intensity value of a sampling point in a circular neighborhood of $P$ sampling points and radius $R$ around $c$ :

$$
g_{p}=I\left(x_{p}, y_{p}\right), \quad p \in\{0,1, \cdots P-1\}
$$


where $x_{p}=x+R \cos (2 \pi p / P)$ and $y_{p}=y-R \sin (2 \pi p / P)$.

Assuming that the local texture $T$ of $c$ is characterized by the joint distribution of intensity values of $P+1$ (where $P>0)$ pixels of $I$ (i.e. $\left.T=t\left(g_{c}, g_{0}, \cdots g_{P-1}\right)\right), g_{c}$ can be subtracted from $g_{p}$ :

$$
T=t\left(g_{c}, g_{0}-g_{c}, \cdots g_{P-1}-g_{c}\right)
$$

Assuming that the central pixel is statistically independent of the differences the distribution can be factorized as $T \approx$ $t\left(g_{c}\right) t\left(g_{0}-g_{c}, \cdots g_{P-1}-g_{c}\right)$.

Since $t\left(g_{c}\right)$ describes the overall intensity over the image, it contains no useful information from the point of view of analyzing local texture patterns, so the local texture can be modelled by $t\left(g_{0}-g_{c}, \cdots g_{P-1}-g_{c}\right)$.

Although these differences are invariant to mean grey level shifts, they are not invariant to other changes, so just the signs of the differences are considered:

$$
T=t\left(s\left(g_{0}-g_{c}\right), \cdots s\left(g_{P-1}-g_{c}\right)\right),
$$

where $s(z)$ is the step function, defined as:

$$
s(z)= \begin{cases}1, & z \geq 0 \\ 0, & z<0\end{cases}
$$

Finally, the local binary pattern operator is derived from this joint distribution by summing the thresholded differences weighted by powers of two. Therefore, the $\operatorname{LBP}_{P, R}$ operator is defined as:

$$
L B P_{P, R}\left(x_{c}, y_{c}\right)=\sum_{p=0}^{P-1} s\left(g_{p}-g_{c}\right) 2^{p}
$$

\subsection{Final image descriptor}

The proposed texture descriptor is built on two steps.

First of all, the GAN-based Minkowski map $\mu_{m}^{h}$ of the different color components $\mathrm{R}, \mathrm{G}$ and $\mathrm{B}$ of the original color image is computed. Thereafter, the $L B P_{P, R}$ operator (eq. 7) of each of these maps is computed, and the three histograms are concatenated. A graphic description of this process is shown in figure 4.

\section{EXPERIMENTS AND RESULTS}

\subsection{Experiments}

\subsubsection{Image database}

The image dataset that have been used in this experiment was composed of 1097 dermoscopic images of pigmented skin lesions, 88 of them being histopathologically confirmed melanomas. Thus, two classes were considered in this experiment: on the one hand confirmed melanomas and on the other hand the remaining benign lesions.

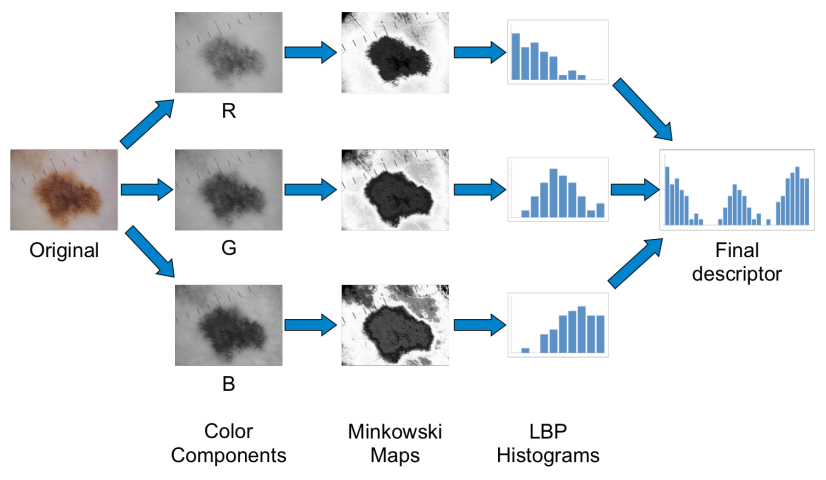

Fig. 4. Image description process.

All images were acquired by several dermatologists equipped by a digital camera (SONY W120) combined with a Heine Delta 20 dermoscope. After the acquisition was carried out, the images were normalized so that they have the same pixel size.

\subsubsection{Image descriptor}

As it was explained in section 2.4, each of the color components R, G and B of the color images of skin lesions has been considered as a grey-level image. First of all, the GANbased Minkowski map area with tolerance 20 was computed from each of them (i.e. $A_{20}^{R}, A_{20}^{G}$ and $A_{20}^{B}$ ). Several values for $m$ were assessed, but the best results were obtained with $m=20$. The other two Minkowski functionals $P$ and $\chi$, as well as different tolerances were assessed, but they yielded worse results. After that, the $\operatorname{LBP}_{P, R}$ was computed from each of these three area maps. The number of samples $P$ was fixed to 8 , but different possible values for the radius $R$ were assessed (specifically, they varied from 1 to 6 ). Thus, the LBP histogram of each component has a length of 256. The final descriptor is a concatenation of the three LBP histograms (i.e. the final descriptor has 768 features).

For the sake of comparison, other descriptor where the $\operatorname{LBP}_{P, R}$ operator was computed directly on the intensities of the color components R, G and B has been assessed.

\subsubsection{Classification}

Images were subsequently classified by means of a feedforward Artificial Neural Network (ANN) working on their feature vectors. The data were normalized before classification, so that they had mean zero and standard deviation one. In this experiment a network with one hidden layer and a logistic sigmoid activation function for the hidden and output layers have been employed. The learning of the network was carried out with the momentum and adaptive learning rate algorithm. Different combinations of training cycles and neurons in the hidden layer have been used, in order to assess the impact of this configuration on the results. Results presented 
Table 1. AUC of the best combination cycles-neurons in the ANN for the descriptors based on the "classical" LBP.

\begin{tabular}{cccc}
\hline R & Num. neurons & Num. cycles & AUC \\
\hline 1 & 10 & 300 & 0.8726 \\
2 & 7 & 300 & $\mathbf{0 . 8 9 4 8}$ \\
3 & 7 & 400 & 0.8934 \\
4 & 5 & 500 & 0.8946 \\
5 & 7 & 400 & 0.8895 \\
6 & 10 & 400 & 0.8898 \\
\hline
\end{tabular}

Table 2. AUC of the best combination cycles-neurons in the ANN for the descriptors based on the GAN-Area-based LBP.

\begin{tabular}{cccc}
\hline $\mathbf{R}$ & Num. neurons & Num. cycles & AUC \\
\hline 1 & 10 & 500 & 0.8547 \\
2 & 10 & 500 & 0.8780 \\
3 & 10 & 500 & 0.8934 \\
4 & 7 & 300 & 0.8976 \\
5 & 10 & 500 & 0.9052 \\
6 & 10 & 500 & $\mathbf{0 . 9 1 1 5}$ \\
\hline
\end{tabular}

in the next section correspond to the best configuration for each approach (classical and adaptive).

The classification was carried out using 10-fold cross validation, and the process was repeated 10 times, in order to avoid possible random effects (e.g. due to the random initialization of the network) and over-fitting. The presented results are an average of these 10 runs.

\subsection{Results}

The receiver operating characteristics (ROC) curve is more suitable to illustrate the performance of a classifier than the accuracy of the classification [14]. It is also widely used in visualizing and analyzing the behavior of diagnostic systems. It depicts relative tradeoffs between benefits (true positives, or sensitivity) and costs (false positives, or 1-specificity). More details about ROC curves can be found in [14].

Tables 1 and 2 show the area under the ROC curve (as a measure of how good this curve is) achieved by the descriptors based on the LBP computed directly on the intensities of the color components and computed on the GAN-Area maps, respectively, depending on the different assessed values of $R$. The tables depict the configuration of cycles-neurons of the network that yielded the best AUC for each value of $R$.

The descriptor based on the LBPs of the GAN-Area maps outperform the classical LBPs with an AUC of 0.9115 when $R=6$ against 0.8948 when $R=2$, respectively. The ROC curves [14] of the classifications corresponding to these configurations are shown in figure 5. In addition, a mean ROC curve, estimated in [5] from the predictions carried out by nine dermatologists with this same image dataset, is also depicted in this figure.

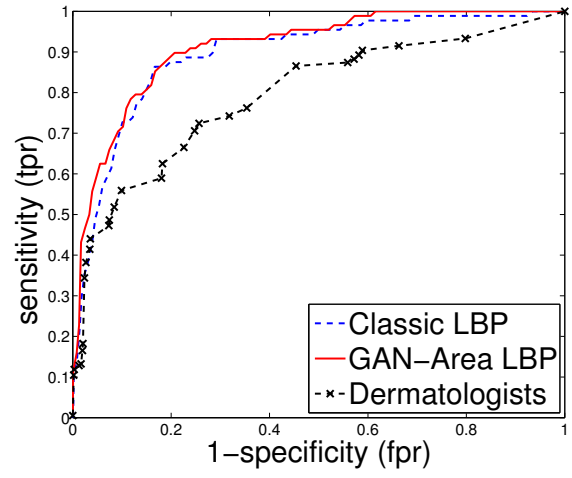

Fig. 5. ROC Curves of the classifiers generated by means of the classical LBPs and the CAN-area-based LBPs, together with the mean ROC curve observed for the dermatologists.

Both the two LBPs descriptors (classical and GAN-based) generated classifiers which outperformed the actual predictions of the dermatologists. In particular, they were found particularly efficient in the distal part of the ROC curves, where the detection of the remaining melanoma is difficult.

\section{CONCLUSIONS AND PERSPECTIVES}

In this work, a texture descriptor has been introduced and applied to the description and classification of color images of nevi as benign lesions or melanoma. The texture descriptor is built from LBPs and local geometrical features.The performance of the proposed descriptor has been evaluated and compared with the classical LBPs and the dermatologists' predictions.

The best results were achieved by the GAN-Minkowskibased LBPs, using the area and a tolerance for the GANs equals to 20. The number of samples of the LBPs was fixed to 8 , and the radius was varied, being the best results achieved for a radius of 6 . This GAN-based approach achieved an AUC of 0.9115, while the classical approach achieved an AUC of 0.8948 in the best configuration of neurons-cycles of the neural network classifier. Both the classic and the adaptive approaches outperformed the dermatologists' predictions on the same image dataset, which obtained mean ROC curve with an AUC of 0.792 [5].

For future work, other GAN-based geometrical and/or morphometrical features [15] could be investigated.

\section{Acknowledgements}

This work has been supported by the project with reference ANR-12-EMMA-0046 from the French National Research Agency. 


\section{REFERENCES}

[1] F. Nachbar, W. Stolz, T. Merkle, A.B. Cognetta, T. Vogt, M. Landthaler, P. Bilek, O. Braun-Falco, and G. Plewig, "The ABCD rule of dermatoscopy: High prospective value in the diagnosis of doubtful melanocytic skin lesions," Journal of the American Academy of Dermatology, vol. 30, no. 4, pp. 551-559, apr 1994.

[2] R.H. Johr, "Dermoscopy: alternative melanocytic algorithms - the ABCD rule of dermatoscopy, menzies scoring method, and 7-point checklist," Clinics in Dermatology, vol. 20, no. 3, pp. 240-247, may 2002.

[3] K. Korotkov and R. Garcia, "Computerized analysis of pigmented skin lesions: A review," Artificial Intelligence in Medicine, vol. 56, no. 2, pp. 69 - 90, 2012.

[4] A. Tenenhaus, A. Nkengne, J.F. Horn, C. Serruys, A. Giron, and B. Fertil, "Detection of melanoma from dermoscopic images of naevi acquired under uncontrolled conditions," Skin research and technology, vol. 16, no. 1, pp. 85-97, 2010.

[5] Y. Wazaefi, S. Paris, and B. Fertil, "Contribution of a classifier of skin lesions to the dermatologist's decision," in Image Processing Theory, Tools and Applications (IPTA), 2012 3rd IEEE International Conference on, Oct 2012, pp. 207-211.

[6] T. Ojala, M. Pietikäinen, and D. Harwood, "A comparative study of texture measures with classification based on featured distributions," Pattern Recognition, vol. 29, no. 1 , pp. $51-59,1996$.

[7] T. Ojala, M. Pietikäainen, and T. Maenpaa, "Multiresolution gray-scale and rotation invariant texture classification with local binary patterns," Pattern Analysis and Machine Intelligence, IEEE Transactions on, vol. 24, no. 7, pp. 971-987, Jul 2002.

[8] M. Pietikäinen, A. Hadid, G. Zhao, and T. Ahonen, Computer Vision Using Local Binary Patterns, Springer, 2011.

[9] J. Debayle and J.-C. Pinoli, "General Adaptive Neighborhood Image Processing: Part I: Introduction and Theoretical Aspects," Journal of Mathematical Imaging and Vision, vol. 25, no. 2, pp. 245-266, aug 2006.

[10] J.-C. Pinoli and J. Debayle, "General adaptive neighborhood mathematical morphology," in Image Processing (ICIP), 2009 16th IEEE International Conference on, 2009, pp. 2249-2252.

[11] K. Michielsen and H. De Raedt, "Integral-geometry morphological image analysis," Physics Reports, vol. 347, no. 6, pp. 461-538, 2001.
[12] K.R. Mecke and D. Stoyan, Statistical Physics and Spatial Statistics, Springer-Verlag Berlin and Heidelberg, 2000.

[13] S. Rivollier, J. Debayle, and J.-C. Pinoli, "Integral geometry and general adaptive neighborhood for multiscale image analysis," International Journal of Signal and Image Processing, vol. 1, no. 3, pp. 141-150, 2010.

[14] T. Fawcett, "An introduction to ROC analysis," Pattern Recognition Letters, vol. 27, no. 8, pp. 861-874, jun 2006.

[15] S. Rivollier, J. Debayle, and J;-C. Pinoli, "Adaptive shape diagrams for multiscale morphometrical image analysis," Journal of Mathematical Imaging and Vision, vol. 49, no. 1, pp. 51-68, 2014. 